\title{
Origin of Unexpected Low Energy Structure in Photoelectron Spectra Induced by Mid-Infrared Strong Laser Fields
}

\author{
Chengpu Liv and Karen Z. Hatsagortsyan \\ Max-Planck-Institut für Kernphysik, Saupfercheckweg 1, D-69117 Heidelberg, Germany
}

(Dated: August 17, 2018)

\begin{abstract}
Using a semiclassical model which incorporates tunneling and Coulomb field effects, the origin of the unexpected low-energy structure (LES) in above-threshold ionization spectrum observed in recent experiments [C. I. Blaga et al., Nature Phys. 5, 335 (2009) and W. Quan et al., Phys. Rev. Lett. 103, 093001 (2009)] is identified. We show that the LES arises due to an interplay between multiple forward scattering of an ionized electron and the electron momentum disturbance by the Coulomb field immediately after the ionization. The multiple forward scattering is mainly responsible for the appearance of LES, while the initial disturbance mainly determines the position of the LES peaks. The scaling laws for the LES parameters, such as the contrast ratio and the maximal energy, versus the laser intensity and wavelength are deduced.
\end{abstract}

PACS numbers: $32.80 . \mathrm{Rm} ; 32.80 . \mathrm{Fb}$

The physics of strong-field photoionization has been extensively studied in the last two decades and is assumed to be well-understood [1] with the three-step model [2] playing a fundamental role. The attosecond science has been developed based on the knowledge how to control the motion of electrons on the atomic scale which paved a way for new methods for measuring molecular structure [3]. The strong field approximation (SFA) [4] serves as an analytical tool to understand the strong-field ionization phenomena which can describe the rescattering via a perturbative approach [1]. Recently, however, a number of strong-field ionization phenomena has been revealed which are caused by non-perturbative influence of the Coulomb field of the atomic core [5]: doublehump and interference structures [6, 7] in the momentum distribution of photoelectrons near ionization threshold, frustrated tunneling ionization [8], and multiphoton assisted recombination [9]. With the advent and further improvement of intense femtosecond mid-infrared laser sources [10], the classical regime of strong-field ionization, when the Keldysh parameter $\gamma \ll 1$ and one expects the SFA to provide an adequate description, has been subjected to more attentive investigation. Here, $\gamma=\sqrt{I_{p} / 2 U_{p}}, I_{p}$ is the ionization potential, and $U_{p}$ the ponderomotive energy. The recent two experiments by C. Blaga et al. [11] and W. Quan et al. [12] on the photoionization of atoms and molecules in strong mid-infrared laser fields reveal a previously unexpected characteristic spike-like low-energy structure (LES) in the energy distribution of electrons emitted along the laser polarization direction, see Fig. 1 (a). These observations manifest a striking contrast to the prediction of the SFA and point to a lack of complete understanding of strong field physics. The numerical solutions of the Schrödinger equation within the single active electron approximation can successfully reproduces the measured LES in the case of any atomic potential. However, the calculations using SFA or SFA with Coulomb corrections [13] fail to describe the LES. Varying the laser polarization from linear to circular, LES is significantly reduced. The latter indicates that forward rescattering [14] is playing an essential role in this process which has been pointed out by Blaga et al. [11] and
Faisal [15]. However, the mechanism which creates the LES remains obscure. Many questions remain unanswered: How exactly does the LES arise? Why does it have a peaked structure? Why is the effect of rescattering more pronounced in mid-infrared laser fields?

In this Letter, we investigate in detail and identify the mechanism of LES. We employ the classical-trajectory Monte Carlo (CTMC) method with tunneling and the Coulomb field of the atomic core fully taken into account. In addition, we provide a qualitative theoretical estimation for the Coulomb field effects: initial Coulomb focusing (CF), multiple forward scattering and asymptotic CF. We quantify their relative role in the electron dynamics and conclude that 1) the behavior of the transverse (with respect to the laser polarization direction) momentum change of the electron due to Coulomb field effects with respect to the ionization phase is the key for understanding of the LES; 2) at mid-infrared wavelengths, multiple scattering of the ionized electron plays a decisive non-perturbative role. In particular, the transverse momentum change of the electron due to multiple scattering distorts the electron phase space to create peaks at low electron energies, while the longitudinal momentum change due to initial $\mathrm{CF}$ shifts the peak energy to higher energies. We investigate and explain qualitatively the scaling of the LES parameters.

In the $\gamma \ll 1$ regime, the electron oscillation amplitude in the laser field $\alpha=E_{0} / \omega^{2}$ exceeds the distance of the electron from the atomic core at the tunnel exit $z_{0}=I_{p} / E_{0}$ : $\alpha / z_{0} \sim 2 / \gamma^{2} \gg 1$ and the transversal distance traveled by the electron during one laser period $x_{0} \sim 2 \pi \sqrt{E_{0}} /\left(2 I_{p}\right)^{1 / 4} \omega$ : $\alpha / x_{0} \sim(1 / 2 \pi) \sqrt{2 I_{p} / \gamma \omega} \gg 1$, where $E_{0}$ and $\omega$ are the laser field amplitude and frequency, respectively (atomic units are used throughout). Therefore, in this regime, the electron travels far from the core during its oscillation in the laser field, and the Coulomb field distorts the electron trajectory only at positions very close to the core. This happens immediately after ionization, corresponding to the initial $\mathrm{CF}$, and when the electron revisits the atomic core and re-scatters. The number of scatterings is large for mid-infrared wavelengths and low-energy photoelectrons [16]: $N_{s} \sim \alpha / x_{0} \sim 10$ at the pa- 

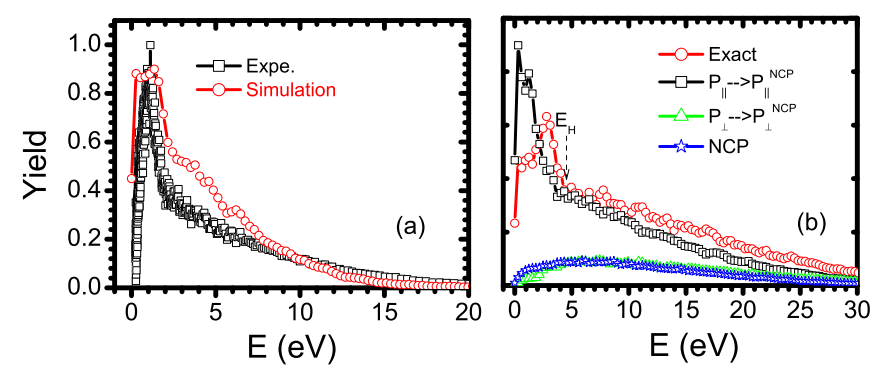

FIG. 1: (color online). Photoelectron spectra (PES): (a) The experimental result (squares) for a Xenon atom in a laser field with peak intensity $I_{0}=3.2 \times 10^{13} \mathrm{~W} / \mathrm{cm}^{2}$ and wavelength $\lambda=2.3 \mu \mathrm{m}$ [11] as well as the corresponding CTMC-simulation (circles). (b) CTMC-simulations for a hydrogen atom with $\lambda=2 \mu \mathrm{m}, I_{0}=9.0 \times$ $10^{13} \mathrm{~W} / \mathrm{cm}^{2}$ : exact (circles); with totally neglecting the Coulomb potential (NCP) (stars); NCP only for the electron longitudinal momenta $P_{\|}$(squares) and NCP only for the transverse momenta $P_{\perp}$ (triangles). The high-energy limit of LES defined by the break in slope is indicated with an arrow.

rameters in [11]. The third Coulomb effect is the asymptotic $\mathrm{CF}$ when the electron momentum is disturbed by the Coulomb field after the laser pulse is switched off. This usually plays an important role for low-energy photoelectrons [8, 17, 18] but is not essential for the LES as shown below.

In our 3D CTMC simulation, the ionized electron wave packet is formed according to the ADK ionization rate [19] and further propagates classically. The electrons are born at the tunnel exit with the following conditions: (i) Along the laser polarization direction, the initial position $z_{i}$ is derived from the effective potential theory [20] and the initial momentum $p_{i \|}=0$ [21]; (ii) The transversal coordinates are $x_{i}=y_{i}=0$. The transverse momentum $p_{i \perp}$ follows the corresponding ADK distribution [19]. The transverse momentum components are $p_{i x}=p_{i \perp} \sin \phi$ and $p_{i y}=p_{i \perp} \cos \phi$, where $\phi$ is the azimuthal angle randomly distributed within an interval of $(0,2 \pi)$. The positions and momenta of electrons after the laser pulse are used to calculate the final asymptotic momenta [18] at the detector. Only electrons emitted along the laser polarization direction within an angle of $\pm 2.5^{\circ}$ are collected. The laser pulse is half-trapezoidal, constant for the first ten cycles and ramped off within the last three cycles. The electrons are launched within the first half cycle $\left(\omega t_{i} \in[0, \pi]\right)$. Our model provides an adequate description of the photoelectron spectrum (PES) and is qualitatively consistent with the experimental results as an example in Fig. 11a) shows.

Now we turn to the clarification of the physical mechanism behind the LES. For simplicity, we consider hydrogen atoms because the atomic structure is not essential for LES. Firstly, we investigate the changes in PES when some factors are neglected, see Fig. 1(b). If the Coulomb potential is neglected after ionization, the LES disappears, as expected. If the Coulomb field effect is neglected only on the longitudinal momentum, the LES shifts towards lower energy and enhances. However, if the Coulomb field effect is neglected only
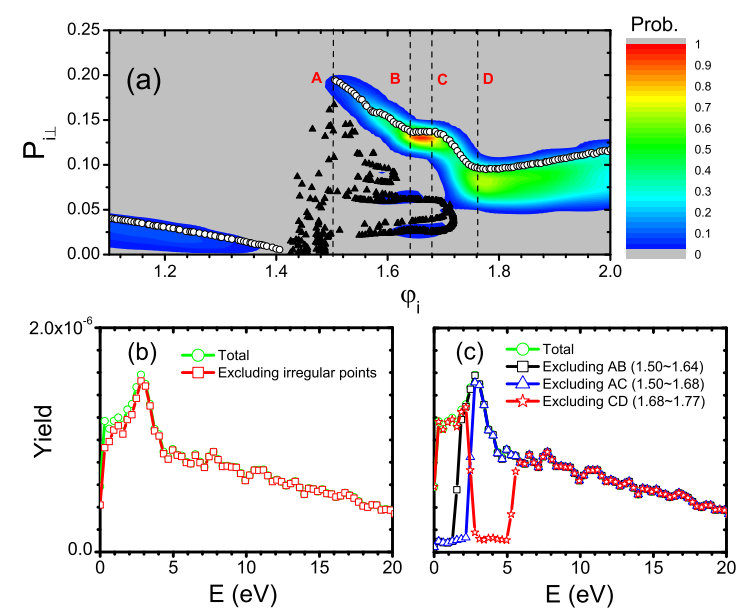

FIG. 2: (color online). (a) The distribution of electrons in the LES in phase space (the initial transverse momentum versus the ionization phase $\left.\varphi_{i} \equiv \omega t_{i}\right)$ within the electron energy interval $(0,20) \mathrm{eV}$, with color coded probability. Irregular points are shown by triangles. The circles indicate the maximum probability for each phase. The laser field is maximal at $\varphi_{i}=\pi / 2$. (b) PES with and without irregular points. (c) PES, the electrons born in the specified phase range are removed. The laser and atom parameters are the same as in Fig. 1(b).

on the transverse momentum, the LES disappears completely. We can deduce that the change of the transverse momentum due to the Coulomb potential is the main source of LES at which we look more closely next.

Secondly, we investigate the phase space distribution of electrons which contribute to the LES, the initial transverse momentum versus the birth phase shown in Fig. 22(a): (i) The electron ionized near the peak of the laser field with a low transverse momentum has a low drift momentum, scatters many times by the atomic core with a low impact parameter and, as a result, shows chaotic behavior (the triangles in Fig. 2 a)). However, the electrons with chaotic dynamics mostly contribute to the high-energy part of PES essential for the formation of a plateau in above-threshold ionization. The contribution of irregular points to the LES amounts only to a few percent [22]. For this reason, when these points are discarded, the LES in PES shows only a little reduction in its lower energy part, see Fig. 2(b). Therefore, the chaotic dynamics is not the cause of the LES. (ii) A large number of electrons with a large initial transverse momentum $\left(p_{i \perp}\right)$ is concentrated after the interaction in the phase space with a low transverse momentum. This is usually termed as CF [23]. The CF is largest near the laser peak and decreases with the increasing of the ionization phase. (iii) More importantly, the decrease of $p_{i \perp}$ is not monotonic but shows a step-like slope change. We state that this slope change is responsible for the appearance of peaks in the LES. In fact, near the points of the slope change of the $p_{i \perp}\left(\varphi_{i}\right)$, the phase space of electrons contributing to the LES (per unit $\varphi_{i}$ ) has local maxima. This can be tested via artificially discarding the electrons between certain characteris- 
tic phases, see Fig. 2(c). The latter shows that the occurrence of the highest part of LES is, in fact, related to the electrons with ionization phases within an interval $(1.64,1.77)$ exactly corresponding to the slope change between $\mathrm{B}$ and $\mathrm{D}$. The behavior of $p_{i \perp}$ versus the ionization phase, in fact, reflects the one of the transverse momentum change due to the Coulomb field $\left(\delta p_{\perp}\right)$, see Fig. 4 (a) below. Then, one needs to explain $\delta p_{\perp}$ non-monotonic behavior with respect to $\phi_{i}$.

Thirdly, we investigate the transverse and longitudinal momentum disturbance of the electron due to the Coulomb field $\left(\delta p_{\perp}\right.$ and $\left.\delta p_{\|}\right)$. For one specific trajectory contributing to LES, $\delta p_{\perp}$ and $\delta p_{\|}$are shown in Fig. 3 a). After the electron's birth, $\delta p_{\perp, \|}$ changes abruptly during a fractional part of the laser period which is known as initial CF [23]. Further momentum change takes place due to scattering when the electron passes the core at the minimum distance $r$ (Fig. 3 b)). We proceed to estimate the contributions of initial CF and multiple scattering, respectively, for electrons with the maximal probability for each phase (circles in Fig. 2(a)). (i) The transverse momentum change due to Coulomb potential $V(r)$ at the sth scattering can be estimated as $\delta p_{\perp}^{(s)} \approx$ $\int \nabla_{\perp} V(r(t)) d t \sim\left(\rho_{s} / r_{s}^{3}\right) \delta t_{s}$, where $r_{s}$ is the distance from the core at the scattering moment, $\rho_{s}$ is the one in the transversal plane, and $\delta t_{s}$ is the scattering duration. When the electron velocity $p_{s}$ at scattering is large, $\delta t_{s} \sim 2 r_{s} / p_{s}$. In the opposite case, $\delta t_{s} \sim 2 \sqrt{2 r_{s} /\left|E\left(\varphi_{s}\right)\right|}$ is determined by the laser field $E\left(\varphi_{s}\right)$ at scattering. Accordingly, $\delta p_{\perp}^{(s)}=-2 \rho_{s} /\left(r_{s}^{2} p_{s}\right)$, if $p_{s}^{2} \gg r_{s}\left|E\left(\varphi_{s}\right)\right|$, or $\delta p_{\perp}^{(s)}=-2^{3 / 2} \rho_{s} / \sqrt{r_{s}^{5}\left|E\left(\varphi_{s}\right)\right|}$, otherwise. $\delta p_{\perp}^{(s)}$ is sensitive to $r_{s}$ and $p_{s}$. Their values for the different scattering events are shown in Figs. 3. (c) and (d). As for the

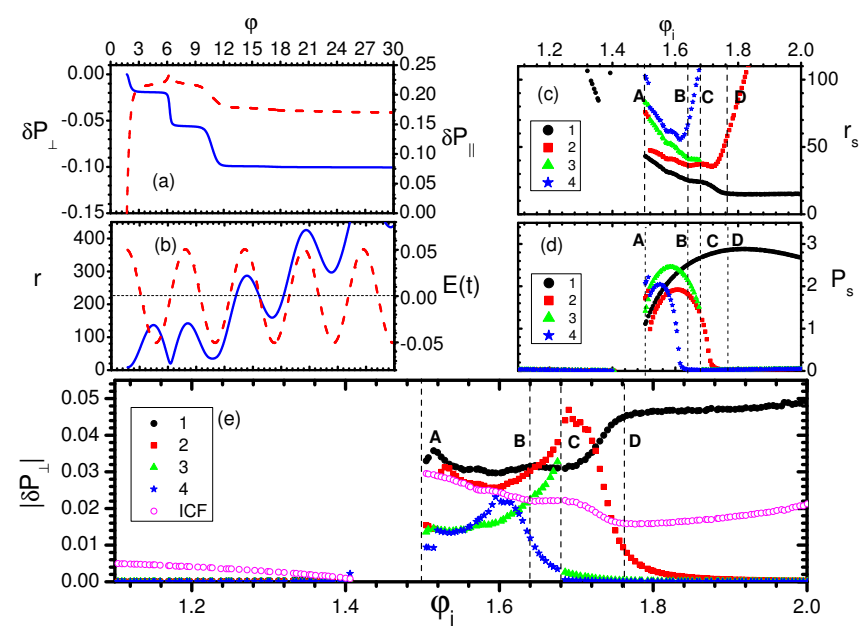

FIG. 3: (color online). (a) The transverse $\delta P_{\perp}$ (solid) and longitudinal $\delta P \|$ (dashed) momentum changes due to the Coulomb field. (b) The distance $r$ (solid) from the core and electric field (dashed) vs. the laser phase for one specific trajectory $\left(\varphi_{i}=1.72\right)$. (c) The position $r_{s}$ and (d) the momentum $p_{s}$ at the sth scattering versus the ionization phase. (e) The estimated $\delta P_{\perp}$ due to different number of scatterings and initial CF. The laser and atom parameters are the same as in Fig. $1(b)$.

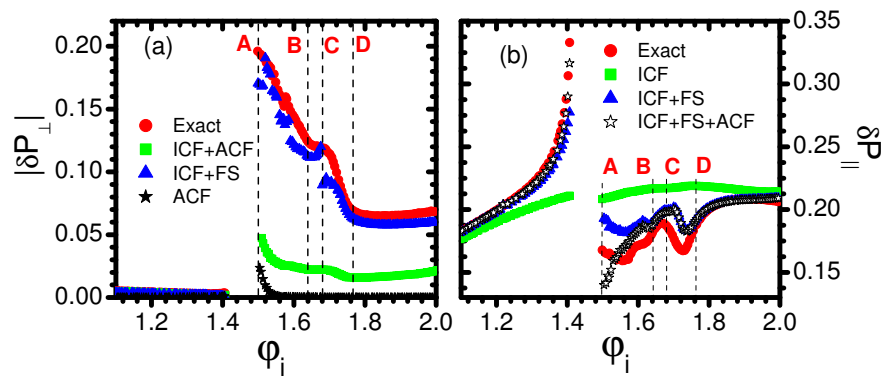

FIG. 4: (color online). The estimation of (a) $\left|\delta P_{\perp}\right|$ and (b) $\delta P_{\|}$described in the text. The contributions of asymptotic $\mathrm{CF}$, the combined contribution of asymptotic and initial $\mathrm{CF}$, and the one of asymptotic $\mathrm{CF}$ and scattering are shown. The laser and atom parameters are the same as in Fig. 1(b).

formula of the $\delta p_{\|}^{(s)}, \rho_{s}$ in the expression for $\delta p_{\perp}^{(s)}$ should be substituted by the absolute value of the scattering coordinate $\left|z_{s}\right|$ along the laser polarization direction. (ii) The transverse momentum change due to initial CF can be estimated as $\delta p_{\perp}^{(I)}=-2 p_{i \perp} f_{\perp}\left(E_{0}, \omega\right)\left|E\left(\varphi_{i}\right)\right| /\left(2 I_{p}\right)^{2}$ and the longitudinal one as $\delta p_{\|}^{(I)}=\pi f_{\|}\left(E_{0}, \omega\right)\left|E\left(\varphi_{i}\right)\right| /\left(2 I_{p}\right)^{3 / 2}$, where the functions $f_{\perp, \|}\left(E_{0}, \omega\right)$ describe the deviation from the simple estimate of [18]. (iii) We estimate the asymptotic CF contribution comparing the asymptotic electron momentum with the one after switching off the laser pulse.

In Fig. 3 (e), the relative contribution of multiple scattering (up to 4th scattering) and initial CF to $\delta p_{\perp}$ is shown calculated using the above formulas. At $\varphi>1.77$ (line D), mainly 1 st scattering and initial CF plays a role. With a little shift of phase towards 1.68 (line $\mathrm{C}$ ), the 2 nd scattering enhances rapidly due to the increase of the scattering energy and the 1st scattering weakens. The significant contribution from the 2 nd scattering results in the slope change between lines $\mathrm{C}$ and $\mathrm{D}$ in Fig. 2 (b). Decreasing the phase further, the 3rd scattering becomes comparable with initial CF, the 1st and 2nd scattering. The competition between 1st-3rd scattering and initial CF results in the flat slope between lines B and C (see Fig. 2 (a)). The increasing contribution of the 1 st-3rd scattering as well as the emerging contribution from the 4th scattering are responsible for the further slope increase after the point B. Summing up our estimations for $\delta p_{\perp}$ and $\delta p_{\|}$due to scattering, initial and asymptotic $\mathrm{CF}$, the exact momentum change is reproduced, see Fig. 4. Neglecting scattering, initial or asymptotic $\mathrm{CF}$, respectively, the contribution of each effect is quantified. The multiple scattering is crucial for the transverse momentum change. It determines the $\delta p_{\perp}\left(\varphi_{i}\right)$ behavior which, in turn, determines the ionization phases corresponding to the LES peaks, and in this way the shape of LES. The initial CF plays a less important role for the transverse momentum change, but a very significant one for the longitudinal momentum change. The latter shifts the LES peak, determining the position of LES. These two points are consistent with the conclusions from Fig. 1(b). The asymptotic CF is only 


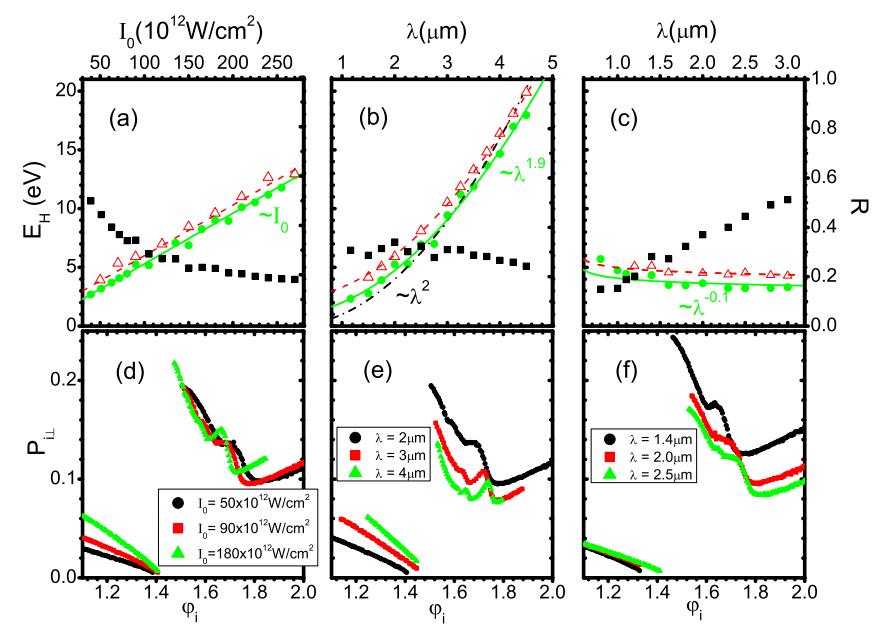

FIG. 5: (color online). The dependence of $E_{H}$ (circles) and $R$ (squares) on (a) laser intensity $I_{0}$ at $\lambda=2 \mu \mathrm{m}$, (b) wavelength $\lambda$ at $I_{0}=9.0 \times 10^{13} \mathrm{~W} / \mathrm{cm}^{2}$ and (c) wavelength $\lambda$ at $\gamma=0.534$. The solid line is a fit to all of the LES data. The triangles and dashed lines are our estimations. In (d), (e) and (f) the electron distribution in phase space for different laser intensities and wavelengths are displayed.

important at near zero energies and has little impact on LES.

Finally, we investigate the dependence of LES on the laser intensity and wavelength, see Fig. 5. Two parameters characterize the LES, the first is $E_{H}$, the high-energy limit of LES [11], as labeled in Fig. 1(b). The second is the contrast ratio $(R)$, the ratio between the integral yield of photoelectrons in the energy interval $\left(0, E_{H}\right)$ and the total ionization yield for electrons emitted along the laser polarization direction. $R$ characterizes the relative height and $E_{H}$ the width of the LES, respectively. $E_{H}$ increases approximately linearly with intensity (see Figs. 5 (a)). Increasing the wavelength, $E_{H}$ slightly deviates from the $\lambda^{2}$-law (i.e. the dependence on only $\gamma$ ) which is mainly due to the increasing initial CF contribution to the $\delta p_{\|}$. The $R$ dependence on the laser parameters is more interesting. The $R$ decreases monotonically with increasing intensity. With increasing wavelength, $R$ first keeps constant and then decreases slowly. The $E_{H}$ and $R$ behavior can be explained qualitatively by inspecting the phase space, see Figs. 5 (d), (e). In Figs. 5(d) and (e), the curves in phase space rotate counter-clockwise resulting in the reduction of electrons within the LES. The reason is the competition between the initial CF and scattering. When fixing the Keldysh parameter (see Fig. 5(c),(f)), the curves in phase space shift parallel to larger phase which yields to the $R$ increase with rising wavelength. At smaller wavelength, $R$, i.e. the LES visibility, becomes smaller. This partly explains why the LES has not been experimentally observed with near infrared lasers. Another reason is the relative suppression of multiple scattering at near infrared wavelength due to quantum effects [22].

In conclusion, the experimental results have been successfully reproduced via the semiclassical calculations and the origin of LES is clarified. At mid-infrared wavelengths the multiple rescattering of ionized electron plays a significant nonper- turbative role which is the main factor creating the LES. The peaks in LES arise due to multiple scattering contributions to the transverse momentum.

We gratefully acknowledge C. I. Blaga and L. F. DiMauro for sharing the experimental data and C. H. Keitel for valuable discussions.

* Electronic address: Chengpu.Liu@ mpi-hd.mpg.de

$\dagger$ Electronic address: k.hatsagortsyan@mpi-hd.mpg.de

[1] W. Becker et al., Adv. Atom. Mol. Opt. Phys. 48, 36 (2000).

[2] P. B. Corkum, Phys. Rev. Lett. 71, 1994 (1993).

[3] M. Lein, J. Phys. B 40, R135 (2007).

[4] L. V. Keldysh, Sov. Phys. JETP 20, 1945 (1964); F. H. M. Faisal, J. Phys. B 6, L89 (1973); H. R. Reiss, Phys. Rev. A 22, 1786 (1980).

[5] S. V. Popruzhenko et al., Phys. Rev. A 77, 053409 (2008); I. A. Burenkov et al., Laser Phys. Lett. 7, 409 (2010).

[6] R. Moshammer et al., Phys. Rev. Lett. 91, 113002 (2003); A. Rudenko et al., J. Phys. B 37, L407 (2004).

[7] A successful quantum mechanical explanation is given by F. H. M. Faisal and G. Schlegel, J. Phys. B. 38, L223 (2005).

[8] T. Nubbemeyer et al., Phys. Rev. Lett. 101, 233001 (2008).

[9] E. S. Shuman et al., Phys. Rev. Lett. 101, 263001 (2008).

[10] C. Erny et al., Appl. Phys. B 96, 257 (2009).

[11] C. I. Blaga et al., Nature Phys. 5, 335 (2009); F. Catoire et al., Laser Phys. 19, 1574 (2009).

[12] W. Quan et al., Phys. Rev. Lett. 103, 093001 (2009).

[13] G. Duchateau et al., Phys. Rev. A 63, 053411 (2001).

[14] The backwards scattering would contribute to the plateau of the PES, see G. G. Paulus et al., Phys. Rev. Lett. 72, 2851 (1994).

[15] F. H. M. Faisal, Nature Phys. 5, 319 (2009).

[16] The number of recollision can be defined as $N_{s} \sim \omega l_{m} / 2 \pi v_{d}$, where $l_{m}$ is the the maximal drift distance and $v_{d}$ the drift velocity. During the drift in the laser polarization direction $v_{d} \sim \sqrt{2 \varepsilon}$ with the electron energy $\varepsilon$ and $l_{m} \sim \alpha$ because the electron passes the origin only when the drift distance is less than $\alpha$. Due to the electron drift in the transversal direction, $v_{d} \sim p_{\perp}$. Accordingly, $N_{s} \sim(\alpha \omega / 2 \pi) \times \min \left\{1 / p_{\perp}, 1 / \sqrt{2 \varepsilon}\right\}=$ $\min \left\{\alpha / x_{0},(1 / 2 \pi) \sqrt{2 U_{p} / \varepsilon}\right\}$.

[17] K. I. Dimitriou et al., Phys. Rev. A 70, 061401(R) (2004).

[18] N. I. Shvetsov-Shilovski et al., Laser Phys. 19, 1550 (2009).

[19] A. M. Perelomov, V. S. Popov, and V. M. Teren'ev, Sov. Phys. JETP 23, 924 (1966); M. V. Ammosov, N. B. Delone, and V. P. Krainov, ibid. 64, 1191 (1986); N. B. Delone and V. P. Krainov, J. Opt. Soc. Am. B 8, 1207 (1991).

[20] L. D. Landau and E. M. Lifshitz, Quantum Mechanics (Pergamon, Oxford, 1977) p. 293.

[21] T. Brabec et al., Phys. Rev. A 54, R2551 (1996).

[22] The contribution to LES of electrons with chaotic dynamics increases with decreasing laser wavelength. However, at rather small laser wavelength, when the impact parameter at first scattering becomes comparable with the electron de Broglie wavelength: $p_{\perp} / \omega \lesssim 1 / \sqrt{2 \varepsilon}$, the classical description fails. Then, the diffraction of the electron wavepacket makes multiple scattering inefficient (see also J. Tate et al., Phys. Rev. Lett. 98, 013901 (2007)) which suppresses the LES. For a LES energy $\varepsilon \approx 3 \mathrm{eV}, p_{\perp}=0.1$, the above condition is $\lambda \lesssim 1 \mu \mathrm{m}$.

[23] G. L. Yudin and M. Y. Ivanov, Phys. Rev. A 63, 033404 (2001); D. Comtois et al., J. Phys. B 38, 1923 (2005); C. Huang et al., Opt. Express 18, 14293 (2010). 